\title{
CONCERNING WILD CANTOR SETS IN $E^{3}$
}

\author{
R. B. $\mathrm{SHER}^{1}$
}

1. Introduction. A well-known example of a wild Cantor set in $E^{3}$ is that described by Antoine in [2]. This example has become known as Antoine's necklace. In this paper we introduce the concept of defining sequences for Cantor sets in $E^{3}$. Using defining sequences, we define a large class of Cantor sets in $E^{3}$, to be referred to as Antoine's necklaces, which are closely related to the original example of Antoine. A necessary and sufficient condition is given for two of these Cantor sets to be equivalently imbedded in $E^{3}$. Finally, we conclude with a result that shows the existence of uncountably many inequivalent upper semicontinuous decompositions of $E^{3}$.

If $A$ is a subset of $E^{3}$, we shall use $\mathrm{Cl} A, \mathrm{Bd} A$, and Int $A$ to denote the closure, boundary and interior, respectively, of $A$. Interior may refer either to the combinatorial interior of a manifold with boundary or to the bounded complementary domain of a compact 2-manifold in $E^{3}$; the correct interpretation will be clear in each case from context.

For terms not defined in this paper (particularly those dealing with linked chains and winding) one may refer to [11].

2. Defining sequences. A sequence $M_{1}, M_{2}, M_{3}, \ldots$ of compact 3-manifolds with boundary is a defining sequence for the Cantor set $M$ in $E^{3}$ if and only if (1) for each positive integer $i, M_{i}$ is the union of a finite number of mutually exclusive polyhedral cubes with handles, (2) for each positive integer $i, M_{i+1} \subset \operatorname{Int} M_{i}$, and (3) $M=\bigcap_{i=1}^{\infty} M_{i}$. One may compare this definition to that of a defining sequence for an upper semicontinuous decomposition of $E^{3}$, [4]. It is known ([3] or [10]) that every Cantor set in $E^{3}$ has a defining sequence. Two such sequences $M_{1}, M_{2}, M_{3}, \cdots$ and $N_{1}, N_{2}, N_{3}, \cdots$ are said to be equivalent if for each positive integer $i$ there is a homeomorphism $h_{i}$ of $E^{3}$ onto itself such that (1) for each positive integer $i, h_{i+1} \mid\left(E^{3}-M_{i}\right)$ $=h_{i} \mid\left(E^{3}-M_{i}\right)$, and (2) for each positive integer $i, h_{i}\left(M_{i}\right)=N_{i}$. It is not difficult to show that this notion yields an equivalence relation on the class of defining sequences of cantor sets in $E^{3}$. We shall use the notation $\left\{M_{i}\right\}$ to denote the defining sequence $M_{1}, M_{2}, M_{3}, \cdots$ and $\left\{M_{i}\right\} \sim\left\{N_{i}\right\}$ to denote that $\left\{M_{i}\right\}$ and $\left\{N_{i}\right\}$ are equivalent defining sequences.

The following theorem may be compared with Theorem 3 of [6].

Received by the editors September 28, 1966.

1 This work was supported by the National Science Foundation Grant GP-6016. 
Theorem 1. The Cantor sets $M$ and $N$ are equivalently imbedded in $E^{3}$ if and only if there exist defining sequences $\left\{M_{i}\right\}$ and $\left\{N_{i}\right\}$ for $M$ and $N$ respectively with $\left\{M_{i}\right\} \sim\left\{N_{i}\right\}$.

Proof. Suppose first of all that $M$ and $N$ are equivalently imbedded in $E^{3}$ and let $h$ be a homeomorphism of $E^{3}$ on to itself carrying $M$ onto $N$.

Let $\left\{M_{i}\right\}$ be a defining sequence for $M$. If for each positive integer $i$ we let $N_{i}=h\left(M_{i}\right)$ and $h_{i}=h$, it is easily seen that $\left\{N_{i}\right\}$ is a defining sequence for $N$ equivalent to $\left\{M_{i}\right\}$.

Now suppose $M$ and $N$ possess equivalent defining sequences $\left\{M_{i}\right\}$ and $\left\{N_{i}\right\}$ respectively, where the equivalence is shown by the sequence of homeomorphisms $\left\{h_{i}\right\}$. We define the function $h$ from $E^{3}$ onto itself as follows: If $x \in E^{3}-M$, let $i$ be an integer such that $x \in M_{i}$ and let $h(x)=h_{i}(x)$. If $x \in M$, let $h(x)=\lim _{i \rightarrow \infty} h_{i}(x)$. The function $h$ so defined is a homeomorphism of $E^{3}$ onto itself and $h(M)=N$.

Unfortunately, each Cantor set in $E^{3}$ has many nonequivalent defining sequences; so one is able to use the above theorem to distinguish between two imbeddings of the Cantor set in $E^{3}$ only if one compares all possible defining sequences for each. In the following section we shall define a collection of Cantor sets, each with a canonical defining sequence which one can use to distinguish imbeddings.

3. Antoine's necklace. The examples described in this section are suggested by the wild Cantor set, of ten referred to as Antoine's necklace, described by Antoine in [2].

Simple closed curves $J_{1}$ and $J_{2}$ in $E^{3}$ are said to be of simple linking type if and only if there is a homeomorphism $h$ of $E^{3}$ onto itself such that $h\left(J_{1}\right)=\left\{(x, y, z) \mid x^{2}+y^{2}=1, z=0\right\}$ and $h\left(J_{2}\right)$ $=\left\{(x, y, z) \mid(y-1)^{2}+z^{2}=1, x=0\right\}$. Note that $J_{1}$ and $J_{2}$ are of simple linking type if and only if $J_{2}$ and $J_{1}$ are of simple linking type. Disjoint solid tori $T_{1}$ and $T_{2}$ in $E^{3}$ are of simple linking type if and only if the centers of $T_{1}$ and $T_{2}$ are of simple linking type. A linked chain of $n \geqq 3$ solid tori is of simple chain type if and only if each linked pair of solid tori in the chain is of simple linking type.

Now, let $T$ be a solid torus in $E^{3}$. Let $Z: T_{1}, \cdots, T_{n}(n \geqq 3)$ be a linked chain of simple chain type in Int $T$ with winding number, $W(Z, T)>0$, and let $M_{1}=\mathrm{U} T_{i}$. Let $Z_{i}: T_{i 1}, \cdots, T_{i n_{i}}\left(n_{i} \geqq 3\right)$ be a linked chain of simple chain type in Int $T_{i}$ with $W\left(Z_{i}, T_{i}\right)>0$ and let $M_{2}=U T_{i j}$. Let $Z_{i j}: T_{i j 1}, \cdots, T_{i j n_{i j}}\left(n_{i j} \geqq 3\right)$ be a linked chain of simple chain type in Int $T_{i j}$ with $W\left(Z_{i j}, T_{i j}\right)>0$, let $M_{3}=\mathrm{U} T_{i j k}$, and so on. This is to be done in such a way that if $d_{i}$ denotes the maximum of the set of diameters of components of $M_{i}$, then $d_{i} \rightarrow 0$ as $i \rightarrow \infty$. Then 
$M=\bigcap_{i=1}^{\infty} M_{i}$ is a Cantor set with defining sequence $\left\{M_{i}\right\}$. Under these conditions, we shall say that $M$ is an Antoine's necklace with canonical defining sequence $\left\{M_{i}\right\}$. The usual Antoine's necklace that has been described in the literature is one in which $n_{i j} \ldots k=4$ and $W\left(Z_{i j} \ldots k, T_{i j \ldots k}\right)=1$.

The Antoine's necklaces have some interesting properties. They are all wildly imbedded in $E^{3}$. If $M$ is an Antoine's necklace with defining sequence $\left\{M_{i}\right\}, i$ is a positive integer, and $J$ is a simple closed curve on $\mathrm{Bd} M_{i}$ which bounds no disk on $\mathrm{Bd} M_{i}$, then $J$ cannot be shrunk to a point in $E^{3}-M$. Also, $M$ is not separated in $E^{3}$ by any 2-sphere.

The following theorem shows that the canonical defining sequences determine the imbedding classes of the Antoine's necklaces. I am told that a similar theorem has also been proven by Professors Steve Armentrout and R. H. Bing.

TheOREM 2. Suppose $M$ and $N$ are Antoine's necklaces in $E^{3}$ with canonical defining sequences $\left\{M_{i}\right\}$ and $\left\{N_{i}\right\}$ respectively. Then $M$ and $N$ are equivalently imbedded in $E^{3}$ if and only if $\left\{M_{i}\right\} \sim\left\{N_{i}\right\}$.

Proof. The "if" portion of the conclusion follows from Theorem 1. Suppose then that $M$ and $N$ are equivalently imbedded in $E^{3}$. It clearly suffices to suppose that $M=N$; that is, that $\left\{M_{i}\right\}$ and $\left\{N_{i}\right\}$ are defining sequences for the same Cantor set. Recalling that $M_{1}$ and $N_{1}$ are polyhedral, we may further suppose that $\mathrm{Bd} M_{1}$ is in general position with respect to $\mathrm{Bd} N_{1}$. This guarantees that $\left(\mathrm{Bd} M_{1}\right) \cap\left(\operatorname{Bd} N_{1}\right)$ is the union of a finite collection of mutually exclusive simple closed curves $J_{1}, \cdots, J_{q}$.

We now describe a sequence of piecewise linear homeomorphisms of $E^{3}$ onto itself. The composition of these homeomorphisms, in the order they are described, will be a homeomorphism $h_{1}$ of $E^{3}$ onto itself which is the identity on $M$ and which carries $M_{1}$ onto $N_{1}$. We first carry $M_{1}$ onto a set whose boundary fails to intersect $\mathrm{Bd} N_{1}$. This is accomplished, in the following paragraph, by removing the simple closed curves $J_{1}, \cdots, J_{q}$ one at a time.

Suppose some $J \in\left\{J_{1}, \cdots, J_{q}\right\}$ bounds a disk on $\operatorname{Bd} N_{1}$. We may choose such a $J$ with the property that if $D$ is the disk on $\mathrm{Bd} N_{1}$ bounded by $J$, then $($ Int $D) \cap\left(\operatorname{Bd} M_{1}\right)=\varnothing$. Since $J$ can be shrunk to a point in $E^{3}-M, J$ must bound a disk, say $D^{\prime}$, on $\mathrm{Bd} M_{1}$. The interior of the 2-sphere $D \cup D^{\prime}$ misses $M$, so there is a homeomorphism of $E^{3}$ onto itself, fixed on $M$, that pushes $D^{\prime}$ onto $D$. Pushing to one side or the other of the component of $\mathrm{Bd} N_{1}$ containing $J$, we adjust $M_{1}$ so that the adjusted $\mathrm{Bd} M_{1}$ intersects $\mathrm{Bd} N_{1}$ in at least one less 
component than the original. Following this procedure, we adjust $M_{1}$ so that the adjusted $\mathrm{Bd} M_{1}$ contains no simple closed curve that bounds a disk on $\left(\mathrm{Bd} M_{1}\right) \cup\left(\mathrm{Bd} N_{1}\right)$. For ease of notation we shall continue to denote the adjusted $M_{1}$ by $M_{1}$. Now let $J$ be one of the remaining elements of $\left\{J_{1}, \cdots, J_{q}\right\}$. There is a $J^{\prime} \in\left\{J_{1}, \cdots, J_{q}\right\}$ such that $J \cup J^{\prime}$ is the boundary of an annulus $A$ on $\mathrm{Bd} N_{1}$ with (Int $A) \subset$ Int $M_{1}$. The simple closed curves $J$ and $J^{\prime}$ lie on the same component, $T$, of $M_{1}$ and Bd $T=A^{\prime} \cup A^{\prime \prime}$ where $A^{\prime}$ and $A^{\prime \prime}$ are annuli bounded by $J \cup J^{\prime}$. The interior of one of the tori $A \cup A^{\prime}$ and $A \cup A^{\prime \prime}$ (say, e.g., $A \cup A^{\prime}$ ) misses $M$. Without disturbing $M$, we push $A$ onto $A^{\prime}$ and then push slightly further to remove $J \cup J^{\prime}$ from (Bd $\left.M_{1}\right) \cap\left(\operatorname{Bd} N_{1}\right)$. Continuing this procedure, we adjust $M_{1}$ so that $\left(\mathrm{Bd} M_{1}\right) \cap\left(\mathrm{Bd} N_{1}\right)=\varnothing$.

Suppose some component, say $T$, of $M_{1}$ lies in Int $N_{1}$ and is nullhomotopic in Int $N_{1}$. Then, any other component of $M_{1}$ linking $T$ must lie in the same component of $N_{1}$ as $T$. But this places all of $M$ in a single component of $N_{1}$, a contradiction. Similarly, no component of $N_{1}$ lying in Int $M_{1}$ is null-homotopic in Int $M_{1}$.

Now suppose some component $T$ of $N_{1}$ contains more than one component of $M_{1}$. The solid torus $T$ is linked by some component of $N_{1}$, hence by some component of $M_{1}$. By the above paragraph, this component of $M_{1}$ links each component of $M_{1}$ in $T$. Hence, there are at least three components of $M_{1}$ each of which links a single component of $M_{1}$. This is a contradiction, since each solid torus in a linked chain links only two elements of the chain. Similarly no component of $M_{1}$ contains more than a single component of $N_{1}$.

We have now established a 1-1 correspondence between components of $M_{1}$ and $N_{1}$. We now show that corresponding components are concentric. Suppose then that $T$ is a component of $M_{1}$ containing in its interior $T^{\prime}$, a component of $N_{1}$. We need only show that $W(C, T)=1$, where $C$ is the center of $T^{\prime}$. Let $T^{\prime \prime}$ be a component of $N_{1}$ so that $T^{\prime}$ and $T^{\prime \prime}$ are of simple linking type. There is a polyhedral disk $D$ with $\mathrm{Bd} D$ a longitudinal simple closed curve of $\mathrm{Bd} T^{\prime \prime}$ and $D \cap C$ a one point set. We may suppose $D$ to be in general position with respect to $\mathrm{Bd} T$. By Theorem 1 of [8], each component of $D \cap \mathrm{Bd} T$ either bounds a disk on Bd $T$, or circles $\mathrm{Bd} T$ once longitudinally and not at all meridionally, or circles Bd $T$ once meridionally and not at all longitudinally. We use a standard cutting off technique to remove simple closed curves on $D$ which bound disks on $\mathrm{Bd} T$. If some component of $D \cap \mathrm{Bd} T$ is longitudinal on $\mathrm{Bd} T$, this component can be shrunk to a point in $E^{3}-C$, a contradiction since Bd $D$ cannot be shrunk to a point in $E^{3}-C$. Hence, the components 
of $D \cap \mathrm{Bd} T$ are meridional on $\mathrm{Bd} T$ and the result is a meridional disk of $T$ which intersects $C$ in a single point. This shows that $W(C, T)=1$. We now complete the construction of $h_{1}$ by shoving $M_{1}$ onto $N_{1}$ using the fact that components of $M_{1}$ and $N_{1}$ lie in concentric pairs.

In a manner similar to the above construction we may construct a homeomorphism $h_{2}$ of $E^{3}$ on to itself which agrees with $h_{1}$ outside of $M_{1}$, which leaves $M$ fixed, and which moves $M_{2}$ onto $N_{2}, \cdots$, etc. This shows that $\left\{M_{i}\right\} \sim\left\{N_{i}\right\}$.

COROLlaRY 1. There are uncountably many inequivalently imbedded Antoine's necklaces in $E^{3}$.

The final results of this section are concerned with the notion of equivalent upper semicontinuous decompositions of $E^{3}$. This concept was first introduced in [6], and the reader will find there the appropriate definitions as well as some related results.

Suppose $G$ is an upper semicontinuous decomposition of $E^{3}$ with a defining sequence $\left\{M_{i}\right\}$, where $\left\{M_{i}\right\}$ satisfies all of the conditions imposed on a canonical defining sequence for an Antoine's necklace except the condition $d_{i} \rightarrow 0$ as $i \rightarrow \infty$. Then $G$ is an Antoine decomposition of $E^{3}$ with canonical defining sequence $\left\{M_{i}\right\}$. An example of an Antoine decomposition of $E^{3}$ appears in [5]. The following theorem follows from the proof of Theorem 2 and Theorem 3 of [6].

Theorem 3. Suppose $G_{1}$ and $G_{2}$ are Antoine decompositions of $E^{3}$ with canonical defining sequences $\left\{M_{i}\right\}$ and $\left\{N_{i}\right\}$. Then $G_{1}$ and $G_{2}$ are equivalent if and only if $\left\{M_{i}\right\}$ and $\left\{N_{i}\right\}$ are equivalent.

Corollary 2. There exist uncountably many inequivalent Antoine decompositions of $E^{3}$.

\section{REFERENCES}

1. M. L. Antoine, Sur la possibilité d'étendre l'homémorphie de deux figures à leur voisinages, C.R. Acad. Sci. Paris 171 (1920), 661-663.

2. - Sur l'homéomor phie de deux figures et de leurs voisinages, J. Math. Pures Appl. 86 (1921), 221-325.

3. S. Armentrout, Decompositions of $E^{3}$ with a compact 0-dimensional set of nondegenerate elements, Trans. Amer. Math. Soc. 123 (1966), 165-177.

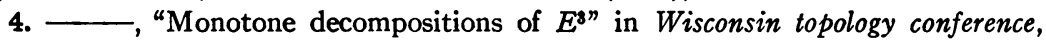
Ann. of Math. Studies No. 60, Princeton, N. J., 1966, pp. 1-25.

5. S. Armentrout and R. H. Bing, $A$ toroidal decomposition of $E^{3}$, Fund. Math. 60 (1967), 81-87.

6. S. Armentrout, L. L. Lininger and D. V. Meyer, "Equivalent decompositions of $E^{8 n}$ in Wisconsin topology conference, Ann. of Math. Studies, No. 60, Princeton, N. J., 1966, pp. 27-31. 
7. R. H. Bing, A homeomorphism between the 3-sphere and the sum of two solid horned spheres, Ann. of Math. (2) 56 (1952), 354-362.

8. - Point-like decompositions of $E^{3}$, Fund. Math. 50 (1962), 437-453.

9. A. Kirkor, Wild 0-dimensional sets and the fundamental group, Fund. Math. 45 (1958), 228-236.

10. H. W. Lambert and R. B. Sher, Point-like 0-dimensional decompositions of $S^{3}$, Pacific J. Math. 24 (1968), 511-518.

11. R. B. Sher, Toroidal decompositions of $E^{3}$, Fund. Math. 61 (1968), 225-241.

The University of Georgia 